\title{
Clinical Evaluation of a Data-Driven Respiratory Gating Algorithm for Whole-Body PET with Continuous Bed Motion
}

\author{
Florian Büther ${ }^{1}$, Judson Jones ${ }^{2}$, Robert Seifert ${ }^{1}$, Lars Stegger ${ }^{1}$, Paul Schleyer ${ }^{2}$, and Michael Schäfers ${ }^{1,3}$ \\ ${ }^{I}$ Department of Nuclear Medicine, University Hospital Münster, Münster, Germany; ${ }^{2}$ Siemens Healthcare, Knoxville, Tennessee; and \\ ${ }^{3}$ European Institute for Molecular Imaging, University of Münster, Münster, Germany
}

\begin{abstract}
Respiratory gating is the standard to prevent respiration effects from degrading image quality in PET. Data-driven gating (DDG) using signals derived from PET raw data is a promising alternative to gating approaches requiring additional hardware (e.g., pressuresensitive belt gating [BG]). However, continuous-bed-motion (CBM) scans require dedicated DDG approaches for axially extended PET, compared with DDG for conventional step-and-shoot scans. In this study, a CBM-capable DDG algorithm was investigated in a clinical cohort and compared with BG using optimally gated (OG) and fully motion-corrected (elastic motion correction [EMOCO]) reconstructions. Methods: Fifty-six patients with suspected malignancies in the thorax or abdomen underwent whole-body ${ }^{18} \mathrm{~F}-\mathrm{FDG}$ CBM PET/ CT using DDG and BG. Correlation analyses were performed on both gating signals. Besides static reconstructions, $O G$ and EMOCO reconstructions were used for $B G$ and DDG. The metabolic volume, SUV max $_{\text {, and }}$ SUV $V_{\text {mean }}$ of lesions were compared among the reconstructions. Additionally, the quality of lesion delineation in the different PET reconstructions was independently evaluated by 3 experts. Results: The global correlation coefficient between BG and DDG signals was $0.48 \pm 0.11$, peaking at $0.89 \pm 0.07$ when scanning the kidney and liver region. In total, 196 lesions were analyzed. SUV measurements were significantly higher in BG-OG, DDG-OG, BG-EMOCO, and DDG-EMOCO than in static images $\left(P<0.001\right.$; median SUV max $_{\text {: }}$ static, $14.3 \pm 13.4$; BG-EMOCO, $19.8 \pm$ 15.7; DDG-EMOCO, $20.5 \pm$ 15.6; BG-OG, $19.6 \pm 17.1$; and DDG$O G, 18.9 \pm 16.6$ ). No significant differences between BG-OG and DDG-OG or between BG-EMOCO and DDG-EMOCO were found. Visual lesion delineation was significantly better in BG-EMOCO and DDG-EMOCO than in static reconstructions $(P<0.001)$; no significant difference was found when comparing $B G$ and DDG for either EMOCO or OG reconstruction. Conclusion: DDG-based motion compensation of CBM PET acquisitions outperforms static reconstructions, delivering qualities comparable to BG approaches. The new algorithm may be a valuable alternative for CBM PET systems.
\end{abstract}

Key Words: PET; PET/CT; respiratory motion; gating; motion correction

J Nucl Med 2020; 61:1520-1527

DOI: $10.2967 /$ jnumed.119.235770

\section{$\mathbf{R}$}

espiratory motion is a source of image degradation in PET and combined PET/CT of the thorax and the abdomen (1), leading

Received Aug. 28, 2019; revision accepted Jan. 30, 2020.

For correspondence or reprints contact: Florian Büther, Department of Nuclear Medicine, University Hospital Münster, Albert-Schweitzer-Campus 1, 48149 Münster, Germany.

E-mail: butherf@uni-muenster.de

Published online Feb. 14, 2020.

COPYRIGHT (C 2020 by the Society of Nuclear Medicine and Molecular Imaging. to effective resolution losses, image blurring, apparent decreased tracer uptake, apparent increased lesion volumes, and potentially lower detection rates for malignancies (2).

Hardware-based gating approaches, using additional equipment to record the respiration of the patient, are widely considered as the reference standard to minimize these effects (3-5). The two most frequently applied systems use sensors measuring pressure changes within a belt around the belly (pressure-sensitive belt gating $[\mathrm{BG}])(6)$ and a camera system monitoring the motion of markers placed on the patient (7).

In contrast, data-driven gating (DDG) approaches derive respiratory waveforms from PET raw data (8). Different DDG methods have been investigated in the past (9-13), and first clinical evaluations have proven their efficacy (14-17). All these studies were performed with limited numbers of bed positions in conventional step-and-shoot mode, in which temporal fluctuations of respiratory frequencies within measured data can be assumed to reflect respiratory motion. However, for wellestablished continuous-bed-motion (CBM) scans (18), this no longer holds true, as the moving bed introduces additional time dependencies.

CBM offers advantages over step-and-shoot scans, such as more uniform axial sensitivities and greater freedom in scanning ranges, as scans are no longer bound to discrete numbers of bed positions (19). CBM also supports different speed profiles, such as by allowing slower scans of regions of higher interest, and does not seem to affect motion correction outcomes compared with step-and-shoot scans (20). Finally, patients seem to prefer CBM over step-and-shoot scans (21). Thus, offering both DDG and CBM seems desirable, potentially combining the advantages of both. However, because of the additional time dependencies, conventional DDG algorithms cannot be expected to work properly per se and needat the least-modifications for CBM. For example, center-ofmass-based algorithms work well with moving tracer accumulations within the scanner (e.g., the heart in ${ }^{18}$ F-FDG PET scans (11)) but would fail when these accumulations enter or leave the field of view during CBM.

In this study, we investigated how a dedicated CBM-DDG algorithm performs in comparison to a conventional BG system. Originally, the first implementation was developed for step-and-shoot acquisitions (10), but implementation was then extended to dynamic PET characterized by nonstationary tracer distributions (22). Prior investigation demonstrated CBM compatibility (23). Additionally, the algorithm potentially avoids the problem of gating signal inversion inherent in DDG in step-and-shoot scans of adjacent bed positions $(24,25)$. Therefore, this study assessed its performance in a 
TABLE 1

Definition of Regions and Correlation Coefficients Between BG and DDG Signals

\begin{tabular}{llllllll}
\hline Parameter & R0 & R1 & R2 & R3 & R4 & R5 & Global \\
\hline Start & Proximal femur & Bladder & Right kidney & Liver dome & Aortic arch & Lung apex & Proximal femur \\
End & Bladder & Right kidney & Liver dome & Aortic arch & Lung apex & Head/neck & Head/neck \\
Correlation & $0.12 \pm 0.17$ & $0.72 \pm 0.15$ & $0.89 \pm 0.07$ & $0.77 \pm 0.16$ & $0.41 \pm 0.32$ & $0.05 \pm 0.08$ & $0.48 \pm 0.11$ \\
coefficient & $(-0.12-0.61)$ & $(0.35-0.96)$ & $(0.68-0.97)$ & $(0.26-0.94)$ & $(-0.71-0.86)$ & $(-0.15-0.21)$ & $(0.30-0.75)$
\end{tabular}

Data are mean $\pm S D$, followed by range in parentheses.

cohort of routine patients undergoing whole-body ${ }^{18}$ F-FDG CBM $\mathrm{PET} / \mathrm{CT}$.

\section{MATERIALS AND METHODS}

\section{Patient Data}

Datasets of 56 patients with suspected malignancies in the thorax or abdomen who underwent ${ }^{18} \mathrm{~F}$-FDG CBM PET/CT scans between December 2018 and July 2019 were included in this analysis. All patients gave written informed consent for retrospective examination. This study was approved by the ethics committee of the Ärztekammer Westfalen-Lippe and the University of Münster (AZ 2019-024-f-S) and was performed in accordance with the 1964 Helsinki declaration and its later amendments.

\section{PET/CT Scan}

The patients fasted overnight before PET/CT. ${ }^{18} \mathrm{~F}-\mathrm{FDG}$ (4 MBq/ $\mathrm{kg}$ of body mass intravenously) was injected about $1 \mathrm{~h}$ before scanning. Patients were then scanned on a Biograph mCT (Siemens Healthcare) with time-of-flight capability and CBM (axial PET field of view, $21.8 \mathrm{~cm}$; spatial resolution at center, $4 \mathrm{~mm}$ in full width at half maximum; sinogram size, $400 \times 168$; number of time-of-flight bins, 13) (26). For scanning, the patients lay supine with the arms above the head. During examination, the BG system AZ-733 V (Anzai Co.) recorded the respiratory signals subsequently used for gating.

Scanning took place from the head or neck to the proximal femur. Based on topograms, low-dose CT scans in end-expiration were obtained (tube voltage, $120 \mathrm{kV}$; effective current, $18 \mathrm{mAs}$; slice thickness, $3.0 \mathrm{~mm}$; duration, 10-20 s), followed by list-mode PET in CBM (free breathing; speed, $1.1 \mathrm{~mm} / \mathrm{s}$; duration, 560-1,270 s).

\section{Gating and Reconstructions}

The investigated DDG algorithm is based on a spectral analysis of PET data (23). These were first projected into a series of 500-ms 3-dimensional volumes by histogramming each prompt event into the voxel corresponding to the center of the time-of-flight bin, on a grid with $32 \times 32$ transaxial voxels, and axially extended to full acquisition length using 2-mm slices. Delayed events do not contain localized motion information and were ignored. The acquisition was then divided into overlapping axial regions of 80-mm length, and the spectral analysis method was used to determine a mask identifying which voxels were subject to respiratory motion. To this end, we defined an initial estimate of respiratory amplitude, $r_{\mathrm{AP}}(t)$, as the change in the anterior-posterior distribution of counts over time. The anteriorposterior distribution at time $t$ is defined as the SD of the corresponding 500-ms 3-dimensional volume, $g_{x y z}(t)$, projected onto the anteriorposterior axis:

$$
r_{\mathrm{AP}}(t)=\mathrm{SD}\left[\sum_{x} \sum_{z} g_{x y z}(t)\right] .
$$

Eq. 1

The respiratory frequency was then defined as

$$
f_{\text {resp }}=\operatorname{argmax}\left\{R_{\mathrm{AP}}(f)\right\}
$$

for all frequencies in the range $0.125 \mathrm{~Hz}<$ $f<0.5 \mathrm{~Hz}$, where $R_{\mathrm{AP}}(f)$ is the power spectrum of $r_{\mathrm{AP}}$ and argmax the function that chooses the argument leading to the maximum value of the respective function. Cardiac frequencies were
FIGURE 1. Comparison of respiratory signals $(A)$ and local correlation coefficients $(B)$ for typical case (landmarks passing scanner center are indicated by arrows). a.u. = arbitrary units. 


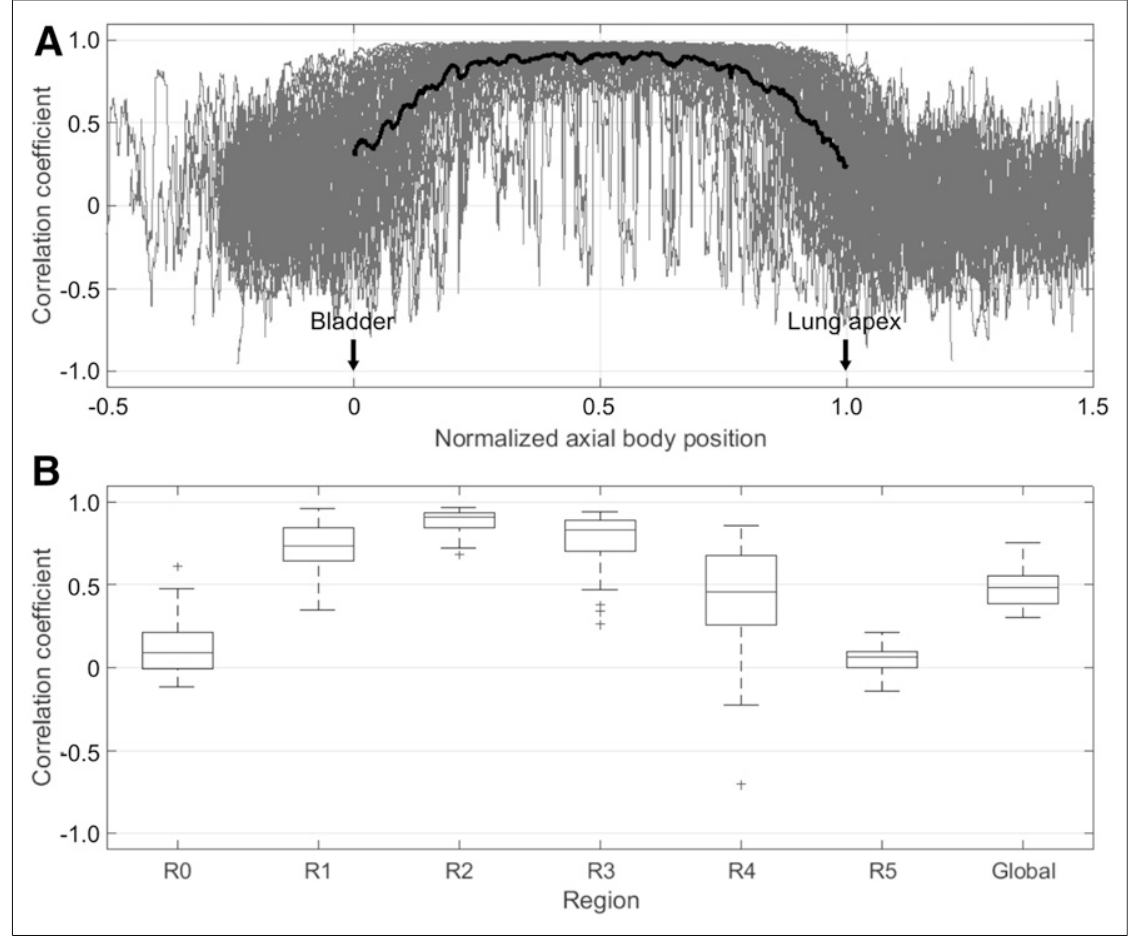

FIGURE 2. (A) Superposition of local correlation coefficients (gray) for all scans in normalized axial position. Average is indicated by black line. (B) Box plot of regional correlation coefficients for all scans. Asterisks denote outliers.

thus excluded. The location $x y z$ for 80 -mm range $n$ was included in the mask $\omega_{x y z, n}$ (i.e., $\omega_{x y z, n} \neq 0$ ) for the corresponding range if

$$
\operatorname{argmax}\left\{G_{x y z, n}(f)\right\}>\text { thresh }
$$

Eq. 3

for frequencies in the range $f_{\text {resp }}-0.05 \mathrm{~Hz}<f_{\text {resp }}<f_{\text {resp }}+0.05 \mathrm{~Hz}$, where $G_{x y z, n}(f)$ is the power spectrum of the 3-dimensional volume at range $n, g_{x y z, n}(t)$ in the temporal domain, and thresh was a threshold determined iteratively such that $10 \%$ of pixels were included in the mask.

A weighting function was required to identify the relative direction that regions of contrast (edges) move (10). By assigning positive or negative weights to mask values from edges that are, respectively, opposed in the direction of motion, the net contribution from moving edges adds constructively to the overall respiratory signal. The weighted mask value at each voxel of region $n$ within the mask was defined as

$$
\omega_{x y z, n}=\cos \left(\Phi_{x y z, n}-\Phi_{\max , n}\right), \quad \text { Eq. } 4
$$

where $\Phi_{x y z, n}$ is the phase angle at $f_{\text {resp }}$, the peak frequency of respiration derived from the spectral analysis, and $\Phi_{\max , n}$ is the mode phase angle of all corresponding voxels in the mask.

Because the relationship between signal gradient sign and the absolute direction of motion is arbitrary and independent for different axial bed positions $(24,25)$, phase angles for region $n$ in CBM acquisitions were offset by the optimal angle $\Phi_{\text {opt }, n}$, which minimized the difference between weights in adjacent, overlapping regions:

$$
\Phi_{\mathrm{opt}, n}=\operatorname{argmin}\left\{\sum_{x, y, z}\left(\Phi_{x y z, n}-\Phi_{x y z, m}\right)^{2}\right\}
$$

with argmin denoting the function that gives the argument leading to the minimum value of the respective function. To initialize Equation $5, \Phi_{\mathrm{opt}, n}$ was set to 0 for region $n=0$, defined as the axially central $80-\mathrm{mm}$ region, and $m$ refers to the adjacent region in the direction of the axial center,

$$
m=\operatorname{sgn}(n)(|n|-1)
$$

Eq. 6

with sgn denoting the sign function. The phase-weighted masks $\omega_{x y z, n}$ from all $80-\mathrm{mm}$ regions were combined into a single mask representing the entire axial extent, $\omega_{x y z, \text { avg }}$, by averaging the nonzero values at $x y z$, that is,

$$
\omega_{x y z, \text { avg }}=\frac{1}{\text { number of } n \text { with } \omega_{x y z, n} \neq 0} \sum_{n} \omega_{x y z, n} .
$$

The respiratory signal $x_{\mathrm{DDG} 0}(t)$ was obtained by summing all values in the masked time series of 3-dimensional volumes, $g_{x y z}(t)$, such that

$$
x_{\mathrm{DDG} 0}(t)=\sum_{x, y, z} \omega_{x y z, \mathrm{avg}} \cdot g_{x y z}(t) .
$$

Eq. 8

Changes in anterior-posterior motion in the volume series were used to ensure that the signal increases and decreases during inspiration and expiration, respectively. Low-frequency changes in signal offset and

TABLE 2

Lesion SUVs and $\mathrm{V}_{50 \%}$ Determined from Static Reconstructions

\begin{tabular}{lccccc}
\hline \multicolumn{1}{c}{ Region } & Lesions $(n)$ & Patients $(n)$ & SUV $_{\text {max }}$ & SUV $_{\text {mean }}$ & $V_{50 \%}\left(\mathrm{~cm}^{3}\right)$ \\
\hline Upper lungs & 26 & 14 & $12.5 \pm 16.1(5.1-84.1)$ & $8.1 \pm 10.3(3.4-52.1)$ & $0.2 \pm 0.7(0.0-2.6)$ \\
Upper mediastinum & 13 & 10 & $18.3 \pm 10.9(5.7-38.9)$ & $12.4 \pm 7.4(3.4-25.9)$ & $0.8 \pm 1.5(0.1-5.4)$ \\
Lower lungs & 82 & 32 & $12.9 \pm 15.8(3.0-88.0)$ & $8.5 \pm 10.9(2.0-58.8)$ & $0.4 \pm 1.7(0.1-11.5)$ \\
Lower mediastinum & 20 & 16 & $14.1 \pm 12.5(5.5-57.4)$ & $9.2 \pm 9.0(3.6-41.9)$ & $0.4 \pm 1.1(0.1-3.6)$ \\
Liver & 31 & 11 & $14.5 \pm 6.0(7.1-26.9)$ & $9.0 \pm 3.8(4.4-17.6)$ & $1.5 \pm 3.8(0.1-14.4)$ \\
Other infradiaphragmatic regions & 24 & 10 & $20.4 \pm 9.3(7.8-43.0)$ & $13.6 \pm 6.1(4.9-28.0)$ & $0.8 \pm 3.0(0.1-12.0)$ \\
Total & 196 & 45 & $14.3 \pm 13.4(3.0-88.0)$ & $9.3 \pm 9.1(2.0-58.8)$ & $0.5 \pm 2.4(0.0-14.4)$
\end{tabular}

Data are median $\pm S D$, followed by range in parentheses. 
amplitude that result from large axial variations in tracer distribution were removed by subtracting a fitted spline,

$$
x_{\mathrm{DDG} 1}(t)=x_{\mathrm{DDG} 0}(t)-\operatorname{spline}(t),
$$

and normalized using a $w=15$-s sliding window,

$$
x_{\mathrm{DDG}}(t)=\frac{x_{\mathrm{DDG} 1}(t)}{\mathrm{SD}\left[x_{\mathrm{DDG} 1}(t-w: t+w)\right]},
$$

where SD is the SD of the window.

Finally, the signals were linearly interpolated to $50 \mathrm{~Hz}$. BG signals $x_{\mathrm{BG}}(t)$ were recorded at $50 \mathrm{~Hz}$.

Both $x_{\mathrm{BG}}(t)$ and $x_{\mathrm{DDG}}(t)$ were corrected for baseline drifts to eliminate nonrespiratory motion. This was done by subtracting a smoothed step function defined on intervals of 15-s length representing the fifth percentile of the original signal. This correction also ensured similar amounts of noise for different axial positions in amplitude-gated CBM images (although potentially resulting in residual blur by noncompensated baseline motion). The optimal gates with $35 \%$ of the measured PET data corresponding to the smallest signal amplitude interval were reconstructed (27).

Additionally, images were reconstructed using an elastic motion correction (EMOCO) algorithm (28). First, motion vectors between optimally gated (OG) and static reconstructions were determined using optical flow estimation. These vectors were then treated as blurring kernels in the forward projection of the reconstruction, effectively deblurring motion effects (29). This algorithm has been validated against an established gate-to-gate motion correction (30).

All datasets were reconstructed using the e7 toolbox (Siemens Healthcare) by ordinary Poisson ordered-subset expectation maximization (3 iterations, 21 subsets) with point-spread function, time of flight, normalization, and random and scatter corrections, resulting in the following reconstructions: static; OG BG and DDG; and EMOCO BG and DDG. Attenuation correction was based on CT data. The
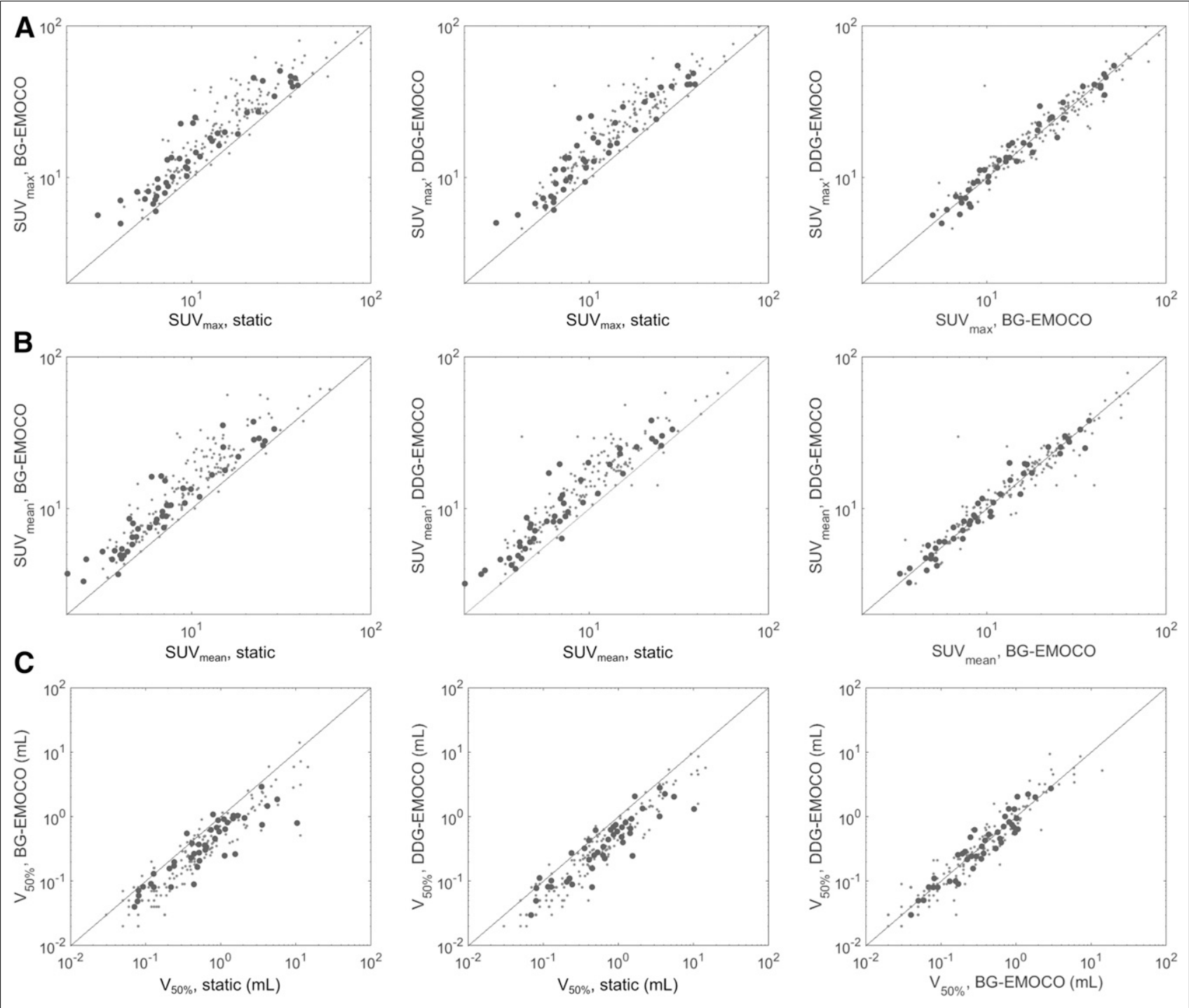

FIGURE 3. Scatterplots of $\mathrm{SUV}_{\max }(\mathrm{A})$, SUV $\mathrm{S}_{\text {mean }}(\mathrm{B})$, and $\mathrm{V}_{50 \%}(\mathrm{C})$ for static, BG-EMOCO, and DDG-EMOCO reconstructions. Subgroup of 1 lesion per scan is denoted by big dots; black line is line of identity. 
images comprised slices of $400 \times 400$ voxels (volume, $2.04 \times 2.04 \times$ $2.03 \mathrm{~mm}$ ). No postreconstruction filter was applied.

\section{Data Analysis}

$x_{\mathrm{BG}}(t)$ and $x_{\mathrm{DDG}}(t)$ were analyzed by calculating the global Pearson correlation coefficient, taking all time indices $t$ into account.

Furthermore, regional correlation coefficients were determined for the following ranges: below the bladder (R0), bladder to right kidney (R1), right kidney to liver dome (R2), liver dome to aortic arch (R3), aortic arch to lung apex (R4), and above the lung apex (R5) (Table 1). The positions of these landmarks were determined from CT, and the times when they were in the center of the PET scanner were calculated.

Finally, local correlation coefficients were calculated for every time $t_{0}$ as the correlation coefficient between $x_{\mathrm{BG}}(t)$ and $x_{\mathrm{DDG}}(t)$ determined for $t_{0}-5 \mathrm{~s}<t<t_{0}+5 \mathrm{~s}$, that is, on a 10-s interval around $t_{0}$, resulting in a better-resolved correlation metric than regional correlation coefficient.

The reconstructed PET images were analyzed for lesions between the bladder and the lung apex. These were characterized individually for all reconstructions by their $\mathrm{SUV}_{\max }$, their apparent metabolic volume based on the respective $50 \% \mathrm{SUV}_{\max }$ threshold $\left(\mathrm{V}_{50 \%}\right)$, and their $\mathrm{SUV}_{\text {mean }}$ in that $\mathrm{V}_{50 \%}$. $\mathrm{SUV}_{\text {max }}$ was determined in a region of interest manually placed over the respective lesion. Since multiple lesions were potentially derived from a scan, 1 lesion per acquisition was randomly chosen, defining a subgroup that was additionally analyzed.

Finally, identically color-scaled coronal slices through the lesions of this subgroup were prepared for an assessment of visual differences between static reconstructions, between BG-EMOCO and DDGEMOCO, and between BG-OG and DDG-OG. These slices were presented independently in random order to 2 nuclear medicine clinicians and 1 medical physicist, who were asked to rate the relative quality in terms of lesion delineation without knowledge of the actual reconstruction method. A relative score was devised as follows: if one method was judged superior to another, +1 was noted; if it was judged inferior, -1 was noted; otherwise, 0 was noted. The scores of the experts were averaged for every comparison and rounded to the nearest integer, resulting in an averaged score per case and comparison.

\section{Statistical Analysis}

Correlation coefficients are given as mean $\pm \mathrm{SD}$. $\mathrm{SUV}_{\text {max }}, \mathrm{SUV}_{\text {mean }}$, and $\mathrm{V}_{50 \%}$ are given as median $\pm \mathrm{SD}$. SUV and $\mathrm{V}_{50 \%}$ differences were tested using 2-tailed Wilcoxon signed-rank tests, and 2-tailed sign ttests were used to assess visual differences (MATLAB, version 2013b; MathWorks). Bonferroni adjustments for pairwise comparisons were applied; the familywise error rate was 0.05 .

\section{RESULTS}

DDG signal calculation typically required $5-10 \mathrm{~min}$ on a $2.4-$ $\mathrm{GHz}, 16$-core, 46-GB random-access-memory system. All 56 signals $x_{\mathrm{DDG}}(t)$ demonstrated typical respiratory features between the bladder and the lung apex; other regions exhibited higher noise levels (Fig. 1A). This finding was corroborated in the correlation analysis between $x_{\mathrm{BG}}(t)$ and $x_{\mathrm{DDG}}(t)$ (Fig. 1B). Generally, despite considerable individual variation, local correlation coefficients demonstrated values close to 0 below the bladder, increasing to 0.9 around the kidneys and liver and decreasing to 0 above the lung apex (Fig. 2A).

Regional correlation coefficients consistently showed the highest values in R2 $(0.89 \pm 0.07)$, decreasing to smaller values in R0 and R5 ( $0.12 \pm 0.17$ and $0.05 \pm 0.08$, respectively); the global correlation coefficient was $0.48 \pm 0.11$ (Fig. 2B; Table 1).
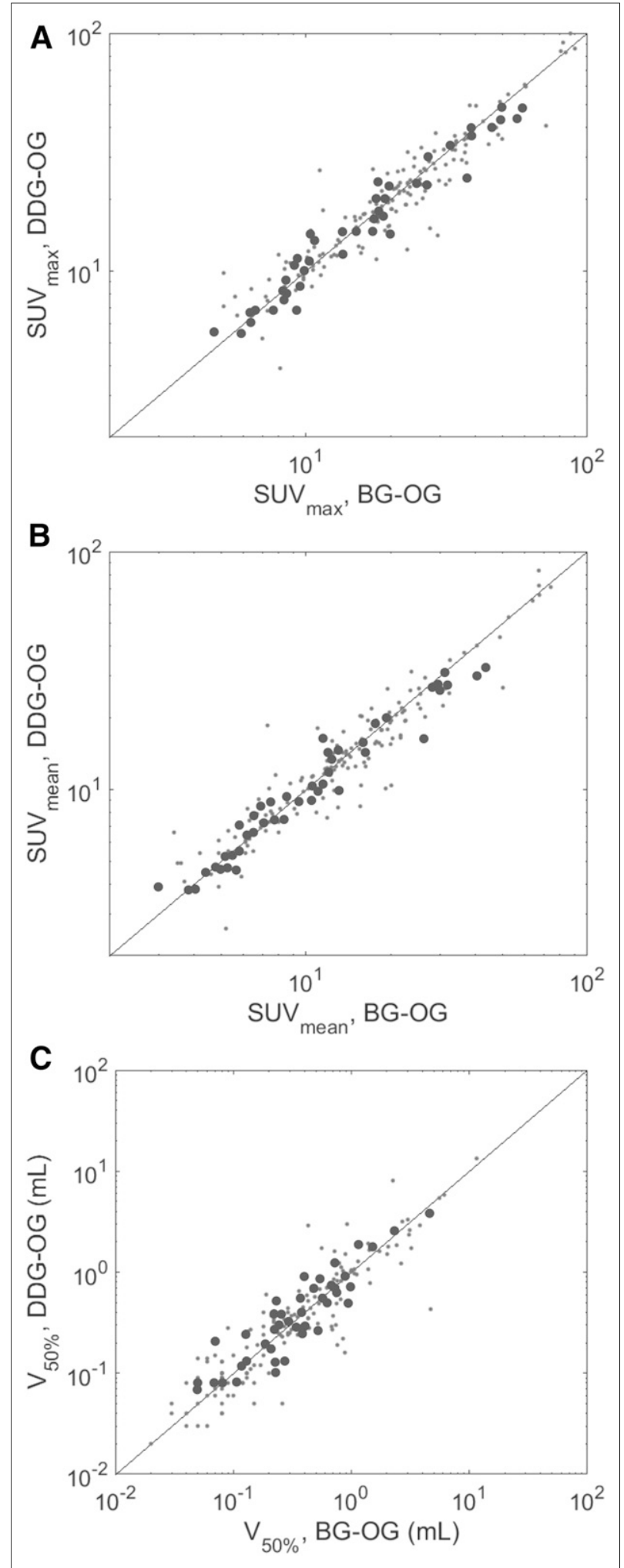

FIGURE 4. Scatterplots of $S U V_{\max }(A)$, SUV mean $(B)$, and $V_{50 \%}(C)$ for $\mathrm{BG}-\mathrm{OG}$, and DDG-OG reconstructions. Subgroup of 1 lesion per scan is denoted by big dots; black line is line of identity.

In total, 196 lesions were analyzed in 45 of the 56 scans (Table 2 ). Twenty-six lesions were located in the upper lungs, 13 in the upper mediastinum, 82 in the lower lungs, 20 in the lower mediastinum, 
TABLE 3

Characteristics of All 196 Lesions for All Correction Strategies

\begin{tabular}{|c|c|c|c|c|c|}
\hline Parameter & Static & BG-EMOCO & DDG-EMOCO & BG-OG & DDG-OG \\
\hline $\mathrm{SUV}_{\max }$ & $14.3 \pm 13.4$ & $19.8 \pm 15.7$ & $20.5 \pm 15.6$ & $19.6 \pm 17.1$ & $18.9 \pm 16.6$ \\
\hline$P$ value to static & & $<0.001$ & $<0.001$ & & \\
\hline$P$ value to $B G$ & & & 0.60 & & 0.19 \\
\hline$S U V_{\text {mean }}$ & $9.3 \pm 9.1$ & $13.5 \pm 11.6$ & $13.7 \pm 11.4$ & $12.6 \pm 12.5$ & $12.4 \pm 12.2$ \\
\hline$P$ value to static & & $<0.001$ & $<0.001$ & & \\
\hline$P$ value to $B G$ & & & 0.65 & & 0.11 \\
\hline$V_{50 \%}\left(\mathrm{~cm}^{3}\right)$ & $0.5 \pm 2.4$ & $0.3 \pm 1.4$ & $0.3 \pm 1.3$ & $0.3 \pm 1.2$ & $0.3 \pm 1.3$ \\
\hline$P$ value to static & & $<0.001$ & $<0.001$ & & \\
\hline$P$ value to $B G$ & & & 0.87 & & 0.50 \\
\hline
\end{tabular}

Data are median \pm SD.

31 in the liver, and 24 in other infradiaphragmatic regions. All lesions were discernible in all reconstructions.

The $\mathrm{SUV}_{\max }$ for all lesions was $14.3 \pm 13.4,19.8 \pm 15.7$, and $20.5 \pm 15.6$ for static reconstructions, BG-EMOCO, and DDGEMOCO, respectively (Figs. 3 and 4; Table 3). The respective $\mathrm{SUV}_{\text {mean }}$ was $9.3 \pm 9.1,13.5 \pm 11.6$, and $13.7 \pm 11.4$, and the respective $\mathrm{V}_{50 \%}$ was $0.5 \pm 2.4,0.3 \pm 1.4$, and $0.3 \pm 1.3 \mathrm{~cm}^{3}$. BGEMOCO and DDG-EMOCO led to values significantly different from the static reconstructions. $\mathrm{SUV}_{\max }$ was $19.6 \pm 17.1$ and $18.9 \pm$ 16.6 for BG-OG and DDG-OG, respectively, and $\mathrm{SUV}_{\text {mean }}$ was $12.6 \pm 12.5$ and $12.4 \pm 12.2$ for BG-OG and DDG-OG, respectively. $\mathrm{V}_{50 \%}$ was $0.3 \pm 1.2 \mathrm{~cm}^{3}$ for BG-OG and $0.3 \pm 1.3 \mathrm{~cm}^{3}$ for DDG-OG. No significant differences between BG and DDG were observed.

$\mathrm{SUV}_{\max }$ in the subgroup of 1 lesion per scan $(n=45)$ was $9.5 \pm$ $10.5,13.4 \pm 13.5$, and 13.5 $\pm 13.7 ; \mathrm{SUV}_{\text {mean }}$ was $6.8 \pm 7.1,8.9 \pm$ 9.4 , and $8.9 \pm 9.2$; and $\mathrm{V}_{50 \%}$ was $0.6 \pm 1.8,0.4 \pm 0.6$, and 0.3 $\pm 0.6 \mathrm{~cm}^{3}$ for static, BG-EMOCO, and DDG-EMOCO images, respectively (Figs. 3 and 4; Table 4). Differences between staticand either of the EMOCO-derived parameters were highly significant. SUV $\mathrm{Sax}_{\max }$ was $15.2 \pm 14.6$ and $14.6 \pm 12.5, \mathrm{SUV}_{\text {mean }}$ was 9.5 \pm 10.2 and $9.0 \pm 8.3$, and $\mathrm{V}_{50 \%}$ was $0.3 \pm 0.8$ and $0.3 \pm 0.7 \mathrm{~cm}^{3}$ for BG-OG and DDG-OG, respectively. Again, no significant differences between BG and DDG were seen.

Visual inspection of the PET images demonstrated improved lesion delineation for both OG and EMOCO compared with static reconstructions, although OG was noticeably noisier (Fig. 5). Quality analysis revealed that static reconstructions were judged superior to BG-EMOCO in a single case, inferior in 33, and equal in 11. Similarly, static reconstructions were judged superior to DDG-EMOCO in no case, inferior in 31 , and equal in 14 . Both BG-EMOCO and DDG-EMOCO were considered significantly superior to static images $(P<0.001$; Fig. 6). No significant difference was seen between BG and DDG: 8 BG-EMOCO cases were superior to DDG-EMOCO, 5 cases were inferior, and 32 cases were the same $(P=0.58)$; DDG-OG was superior to BG-OG in 10 cases, inferior in 6 , and equal in 29 ( $P=0.45$; Fig. 6).

\section{DISCUSSION}

First clinical studies already have demonstrated that DDG methods are equivalent to BG methods in terms of image quality and lesion quantification (14,15); accordingly, vendors have started incorporating DDG algorithms into their PET systems

TABLE 4

Characteristics of 45 Randomly Chosen Lesions for All Correction Strategies

\begin{tabular}{|c|c|c|c|c|c|}
\hline Paramete & Static & BG-EMOCO & DDG-EMOCO & BG-OG & DDG-OG \\
\hline SUV $_{\max }$ & $9.5 \pm 10.5$ & $13.4 \pm 13.5$ & $13.5 \pm 13.7$ & $15.2 \pm 14.6$ & $14.6 \pm 12.5$ \\
\hline$P$ value to static & & $<0.001$ & $<0.001$ & & \\
\hline$P$ value to $B G$ & & & 0.21 & & 0.28 \\
\hline$S U V_{\text {mean }}$ & $6.8 \pm 7.1$ & $8.9 \pm 9.4$ & $8.9 \pm 9.2$ & $9.5 \pm 10.2$ & $9.0 \pm 8.3$ \\
\hline$P$ value to static & & $<0.001$ & $<0.001$ & & \\
\hline$P$ value to $B G$ & & & 0.44 & & 0.20 \\
\hline$V_{50 \%}\left(\mathrm{~cm}^{3}\right)$ & $0.6 \pm 1.8$ & $0.4 \pm 0.6$ & $0.3 \pm 0.6$ & $0.3 \pm 0.8$ & $0.3 \pm 0.7$ \\
\hline$P$ value to static & & $<0.001$ & $<0.001$ & & \\
\hline$P$ value to $B G$ & & & 0.71 & & 0.33 \\
\hline
\end{tabular}

Data are median \pm SD. 


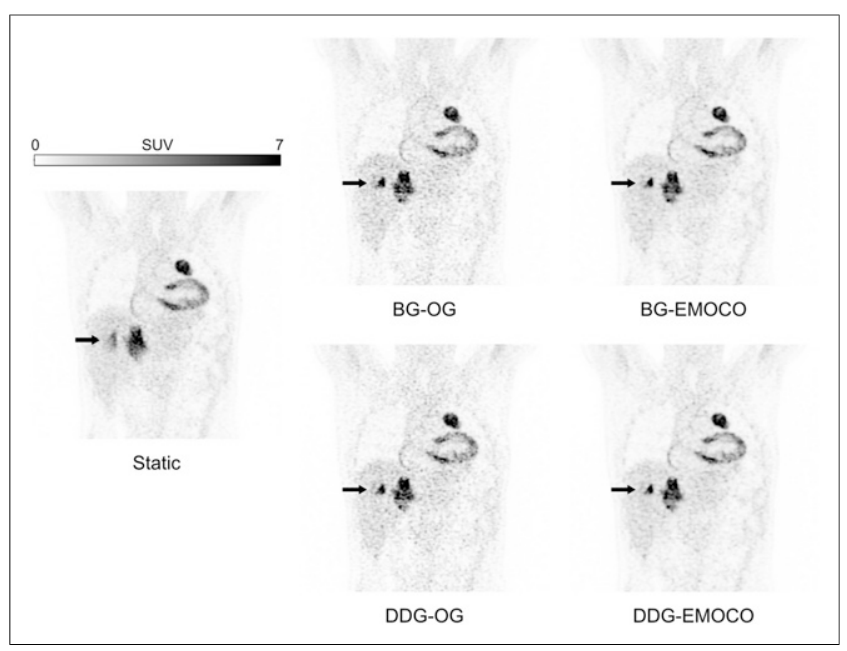

FIGURE 5. Typical outcome of OG and EMOCO using BG and DDG signals, compared with static reconstruction. Visual improvements of liver lesions (arrows) are apparent.

(17). However, more studies are needed to ensure that DDG works well in a wide range of applications, radiotracers, and scanning modes (31).

In this respect, our study closes a gap by evaluating a novel CBM-DDG method in a larger patient cohort undergoing wholebody PET. In general, this algorithm resulted in motion-compensated PET images with quality and accuracy comparable to conventional methods, exploiting the advantages of both CBM and DDG in routine scans. An independent study using a similar algorithm but a smaller number of datasets $(n=15)$ already has demonstrated comparable results, with average regional correlation coefficients of $0.15,0.69,0.82,0.75,0.46$, and 0.07 for regions R0, R1, R2, R3, R4, and R5, respectively (32). However, SUV seemed to be smaller for DDG than for BG, contrasting our results without significant differences.

Some results of our study are of particular interest. The comparison between $x_{\mathrm{BG}}(t)$ and $x_{\mathrm{DDG}}(t)$ demonstrated considerable correlation even in deep abdominal regions (R0 and R1) in some scans,

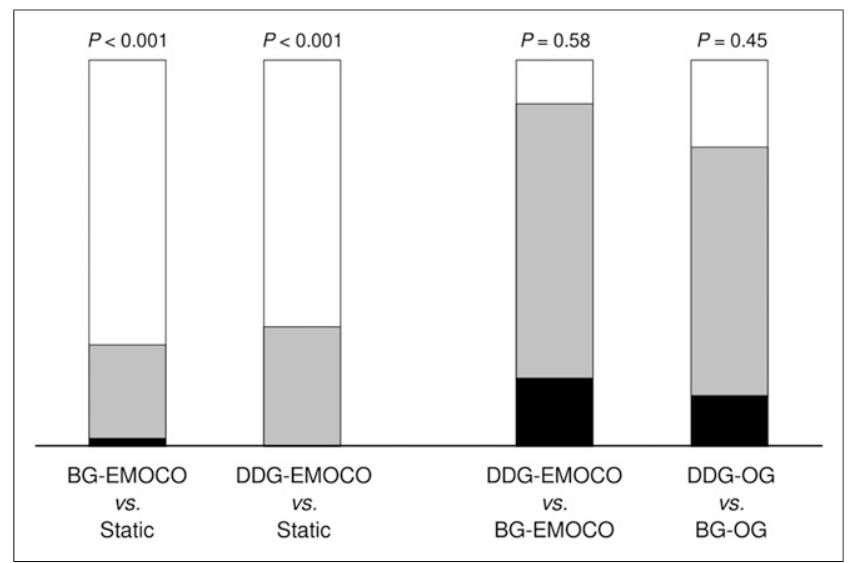

FIGURE 6. Pairwise visual quality comparison between EMOCO and static reconstructions (left) and between DDG and BG (right) ( $n=45)$. White areas denote cases in which method 1 was superior to method 2; black areas denote cases in which method 1 was inferior; and gray areas denote cases in which quality was equal. indicating the presence of respiratory motion there. Unfortunately, because only 4 lesions were detected in R1 in our cohort, no general conclusion can be drawn from the SUV analysis. However, some patients had elevated ${ }^{18}$ F-FDG uptake in several colon segments; these were visually better defined in both BG- and DDGderived images than in static images (Fig. 7). A comparison with observations by Walker et al. confirms that respiratory motion plays a significant role in these regions, as these investigators found respiratory information in raw data of regions up to 40 $\mathrm{cm}$ below the liver dome (17).

In general, good correlations in $x_{\mathrm{BG}}(t)$ and $x_{\mathrm{DDG}}(t)$ were found between the bladder and the lung apex, making possible an imaging workflow that automatically corrects for motion between these boundaries. Decreases in correlation from the lung base to the apex can be explained by smaller respiratory shifts measured by the DDG algorithm toward the apex. This raises the question of whether motion resolution in the upper lung is still sufficient to compete with BG. Surprisingly, an analysis of the lesions located in $\mathrm{R} 4(n=39)$ revealed a slightly larger median $\mathrm{SUV}_{\max }$ and $\mathrm{SUV}_{\text {mean }}$ and a smaller $\mathrm{V}_{50 \%}$ for DDG than for BG (e.g., $S \mathrm{SV}_{\text {max }}$ : $17.1 \pm 15.9$ vs. $17.8 \pm 15.4$ for BG-EMOCO and DDG-EMOCO, respectively). However, this difference was not statistically significant (with pre-Bonferroni $P$ values of $0.05-0.11$ for SUV). Larger cohorts are needed to analyze DDG accuracy in the upper lungs. Nevertheless, this possibility would be in line with some observations of hysteresis effects leading to phase differences in respiration between different body regions (33).

Our study had some additional limitations. First, only ${ }^{18}$ F-FDG PET/CT datasets were included. Although ${ }^{18} \mathrm{~F}-\mathrm{FDG}$ is still the radiotracer used most often in clinical scans, other tracers, such as ligands of the prostate-specific membrane antigen, are becoming increasingly popular for PET imaging. It remains to be seen how DDG performs in these scans.

Furthermore, the observed increases in SUV and decreases in $\mathrm{V}_{50 \%}$ were apparently larger than in similar studies $(15,34)$. This finding highlights that lesion quantification is dependent on many factors, especially reconstruction parameters and spatial resolution. We chose to maximize image resolution in order to detect even small differences in performance between BG and DDG. Thus, we did not apply postreconstruction filters, and we reconstructed on a high-resolution grid, leading to higher SUV increases and $\mathrm{V}_{50 \%}$ decreases. These values may not represent typical clinical

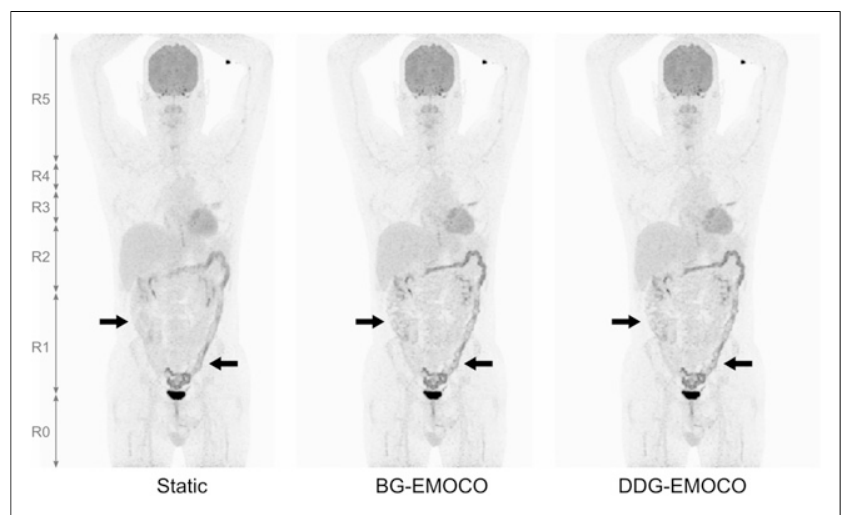

FIGURE 7. Maximum-intensity projection of scan with elevated colon uptake (arrows; R1 and R2) demonstrating better delineation with BG and DDG. 
outcomes; they rather reflect maximal differences in SUV and $\mathrm{V}_{50 \%}$ achieved by gating and motion correction within the settings of this study.

\section{CONCLUSION}

The investigated DDG algorithm generated accurate respiratory signals from CBM PET raw data. Gated and motion-corrected images were comparable to a conventional BG approach in terms of lesion quantification and visual quality. The proposed DDG method seems to be a promising motion correction alternative for routine whole-body CBM PET scans.

\section{DISCLOSURE}

Florian Büther and Michael Schäfers received project funding by Siemens Healthcare GmbH, Erlangen, Germany. Judson Jones and Paul Schleyer are employees of Siemens Healthcare GmbH, Knoxville, TN. Florian Büther, Robert Seifert, Lars Stegger, and Michael Schäfers have control of any data that might present a conflict of interest for employees or consultants. No other potential conflict of interest relevant to this article was reported.

\section{ACKNOWLEDGMENTS}

We thank Erika Düx, Umi Kim-Yeo, and Dr. Benjamin Noto for their excellent support during the study.

\section{KEY POINTS}

QUESTION: Is DDG as accurate as BG approaches in CBM PET?

PERTINENT FINDINGS: This study demonstrated no significant difference in lesion quantification or visual quality between DDG and $B G$, and both methods outperformed static reconstructions.

IMPLICATIONS FOR PATIENT CARE: The presented DDG method for CBM may replace BG approaches, leading to simplifications in workflow and patient management.

\section{REFERENCES}

1. Liu C, Pierce LA, Alessio AM, Kinahan PE. The impact of respiratory motion on tumor quantification and delineation in static PET/CT imaging. Phys Med Biol. 2009;54:7345-7362.

2. Polycarpou I, Tsoumpas C, King AP, Marsden PK. Impact of respiratory motion correction and spatial resolution on lesion detection in PET: a simulation study based on real MR dynamic data. Phys Med Biol. 2014;59:697-713.

3. Guerra L, Ponti E, Morzenti S, et al. Respiratory motion management in PET/ CT: applications and clinical usefulness. Curr Radiopharm. 2017;10:85-92.

4. Frood R, McDermott G, Scarsbrook A. Respiratory-gated PET/CT for pulmonary lesion characterization: promises and problems. Br J Radiol. 2018;91:20170640.

5. Pan T. Respiratory gating in PET/CT: a step in the right direction. J Nucl Cardiol. 2018;25:416-418.

6. Grootjans W, de Geus-Oei LF, Meeuwis AP, et al. Amplitude-based optimal respiratory gating in positron emission tomography in patients with primary lung cancer. Eur Radiol. 2014;24:3242-3250.

7. Lupi A, Zaroccolo M, Salgarello M, et al. The effect of ${ }^{18}$ F-FDG-PET/CT respiratory gating on detected metabolic activity in lung lesions. Ann Nucl Med. 2009;23:191-196.

8. Kesner AL, Schleyer PJ, Büther F, et al. On transcending the impasse of respiratory motion correction applications in routine clinical imaging: a consideration of a fully automated data driven motion control framework. EJNMMI Phys. 2014;1:8.

9. He J, O'Keefe GJ, Gong SJ, et al. A novel method for respiratory motion gating with geometric sensitivity of the scanner in 3D PET. IEEE Trans Nucl Sci. 2008;55:2557-2565.
10. Schleyer PJ, O'Doherty MJ, Barrington SF, Marsden PK. Retrospective datadriven respiratory gating for PET/CT. Phys Med Biol. 2009;54:1935-1950.

11. Büther F, Dawood M, Stegger L, et al. List mode-driven cardiac and respiratory gating in PET. J Nucl Med. 2009;50:674-681.

12. Kesner AL, Kuntner C. A new fast and fully automated software based algorithm for extracting respiratory signal from raw PET data and its comparison to other methods. Med Phys. 2010;37:5550-5559.

13. . Thielemans K, Rathore S, Engbrant F, Razifar P. Device-less gating for PET/CT using PCA. In: 2011 IEEE Nuclear Science Symposium Conference Record. Piscataway, NJ: IEEE; 2011:3904-3910.

14. Kesner AL, Chung JH, Lind KE, et al. Validation of software gating: a practical technology for respiratory motion correction in PET. Radiology. 2016;281:239248.

15. Büther F, Vehren T, Schäfers KP, Schäfers M. Impact of data-driven respiratory gating in clinical PET. Radiology. 2016;281:229-238.

16. Walker MD, Bradley KM, McGowan DR. Evaluation of principal component analysis-based data-driven respiratory gating for positron emission tomography. Br J Radiol. 2018;91:20170793.

17. Walker MD, Morgan AJ, Bradley KM, McGowan DR. Evaluation of data-driven respiratory gating waveforms for clinical PET imaging. EJNMMI Res. 2019;9:1.

18. Osborne DR, Acuff S, Cruise S, et al. Quantitative and qualitative comparison of continuous bed motion and traditional step and shoot PET/CT. Am J Nucl Med Mol Imaging. 2014;5:56-64.

19. Panin VY, Smith AM, Hu J, et al. Continuous bed motion on clinical scanner: design, data correction, and reconstruction. Phys Med Biol. 2014;59:6153-6174.

20. Meier JG, Erasmus JJ, Gladish GW, Peterson CB, Diab RH, Mawlawi OR. Characterization of continuous bed motion effects on patient breathing and respiratory motion correction in PET/CT imaging. J Appl Clin Med Phys. 2020;21: $158-165$.

21. Schatka I, Weiberg D, Reichelt S, et al. A randomized, double-blind, crossover comparison of novel continuous bed motion versus traditional bed position whole-body PET/CT imaging. Eur J Nucl Med Mol Imaging. 2016;43:711-717.

22. Schleyer PJ, Thielemans K, Marsden PK. Extracting a respiratory signal from raw dynamic PET data that contain tracer kinetics. Phys Med Biol. 2014;59:43454356.

23. Schleyer PJ, Hong I, Jones J, et al. Data-driven respiratory gating whole body PET using continuous bed motion. In: 2018 IEEE Nuclear Science Symposium and Medical Imaging Conference Proceedings (NSS/MIC). Piscataway, NJ: IEEE; 2018:1-5.

24. Schleyer PJ, O'Doherty MJ, Marsden PK. Extension of a data-driven gating technique to 3D, whole body PET studies. Phys Med Biol. 2011;56:3953-3965.

25. Bertolli O, Arridge S, Wollenweber SD, et al. Sign determination methods for the respiratory signal in data-driven PET gating. Phys Med Biol. 2017;62:32043220 .

26. Jakoby BW, Bercier Y, Conti M, et al. Physical and clinical performance of the mCT time-of-flight PET/CT scanner. Phys Med Biol. 2011;56:2375-2389.

27. van Elmpt W, Hamill J, Jones J, et al. Optimal gating compared to 3D and 4D PET reconstruction for characterization of lung tumours. Eur J Nucl Med Mol Imaging. 2011;38:843-855.

28. Hong I, Jones J, Hamill J, et al. Elastic motion correction for continuous bed motion whole-body PET/CT. In: 2016 IEEE Nuclear Science Symposium, Medical Imaging Conference and Room-Temperature Semiconductor Detector Workshop (NSS/MIC/RTSD). Piscataway, NJ: IEEE; 2016:1-2.

29. Hong I, Jones J, Casey M. Ultrafast elastic motion correction via motion deblurring. In: 2014 IEEE Nuclear Science Symposium and Medical Imaging Conference (NSS/MIC). Piscataway, NJ: IEEE; 2014;1-2.

30. Pösse S, Hong I, Mannweiler D, et al. Respiratory motion compensation in PET/ $\mathrm{CT}$ : evaluation of an elastic motion compensation technique based on motion deblurring [abstract]. Nucl Med (Stuttg). 2018;57(suppl):A52.

31. Kesner A, Ross Schmidtlein C, Kuntner C. Real-time data-driven motion correction in PET. EJNMMI Phys. 2019;6:3.

32. Meier J, Schleyer PJ, Judson J, et al. Evaluation of a novel data driven gating technique for PET/CT imaging with continuous bed motion [abstract]. $\mathrm{J} \mathrm{Nucl}$ Med. 2019;60(suppl 1):523.

33. Dasari PKR, Shazeeb MS, Könik A, et al. Adaptation of the modified Bouc-Wen model to compensate for hysteresis in respiratory motion for the list-mode binning of cardiac SPECT and PET acquisitions: Testing using MRI. Med Phys. 2014;41:112508.

34. Werner MK, Parker JA, Kolodny GM, et al. Respiratory gating enhances imaging of pulmonary nodules and measurement of tracer uptake in FDG PET/CT. AJR. 2009;193:1640-1645. 